TRANSACTIONS OF THE

AMERICAN MATHEMATICAL SOCIETY

Volume 360, Number 12, December 2008, Pages 6371-6387

S 0002-9947(08)04446-2

Article electronically published on July 28, 2008

\title{
POLYHEDRAL REALIZATION OF THE HIGHEST WEIGHT CRYSTALS FOR GENERALIZED KAC-MOODY ALGEBRAS
}

\author{
DONG-UY SHIN
}

\begin{abstract}
In this paper, we give a polyhedral realization of the highest weight crystals $B(\lambda)$ associated with the highest weight modules $V(\lambda)$ for the generalized Kac-Moody algebras. As applications, we give explicit descriptions of crystals for the generalized Kac-Moody algebras of ranks 2, 3, and Monster algebras.
\end{abstract}

\section{INTRODUCTION}

The quantum groups introduced by Drinfel'd and Jimbo, independently, are deformations of the universal enveloping algebras of Kac-Moody algebras [5, 6]. More precisely, let $\mathfrak{g}$ be a Kac-Moody algebra and $U(\mathfrak{g})$ be its universal enveloping algebra. Then, for each generic parameter $q$, we associate a Hopf algebra $U_{q}(\mathfrak{g})$, called the quantum group, whose structure tends to that of $U(\mathfrak{g})$ as $q$ approaches 1. The important feature of quantum groups is that the representation theory of $U_{q}(\mathfrak{g})$ is the same as that of $U(\mathfrak{g})$. Therefore, to understand the structure of representations over general quantum groups $U_{q}(\mathfrak{g})$, it is enough to understand that of representations over $U_{q}(\mathfrak{g})$ for some special parameter $q$ which is easy to treat. The crystal bases, introduced by Kashiwara [12, 13, can be viewed as bases at $q=0$ for the integrable modules over quantum groups. They give a structure of colored oriented graphs, called the crystal graphs, reflecting the combinatorial structure of integrable modules. For instance, one of the major goals in representation theory is to find an explicit expression for the characters of representations, and this can be obtained by finding an explicit combinatorial description of crystal bases. So one of the most fundamental problems in crystal basis theory is to construct the crystal basis explicitly. In order to answer this, several kinds of combinatorial objects have been invented [10, 11, 15, 16].

In 14, Kashiwara introduced the embedding of crystals $\Psi_{\iota}: B(\infty) \hookrightarrow \mathbf{Z}^{\infty}$, where $\iota$ is some infinite sequence from the index set of simple roots. But, in general, it is not easy to find the image $\operatorname{Im} \Psi_{\iota}$. In 3, Cliff described the image of the Kashiwara embedding for the classical Lie algebras and some reduced expression. For more general types, Zelevinsky and Nakashima obtained the exact image of the

Received by the editors December 11, 2005 and, in revised form, November 8, 2006.

2000 Mathematics Subject Classification. Primary 81R50; Secondary 17 B37.

Key words and phrases. Highest weight crystals, generalized Kac-Moody algebras, Monster algebras.

This research was supported by the research fund of Hanyang University (HY-2007-000-0000$5889)$.

(C)2008 American Mathematical Society Reverts to public domain 28 years from publication 
embedding by a unified method, called the polyhedral realization [18. Moreover, in [17, Nakashima introduced the crystal $R_{\lambda}=\left\{r_{\lambda}\right\}$, and he showed that the connected component containing $u_{\infty} \otimes r_{\lambda}$ is isomorphic to the highest weight crystal $B(\lambda)$, where $u_{\infty}$ is the highest weight vector in $B(\infty)$. Applying this property to the Kashiwara embedding, he gave the embedding of crystals $\Psi_{\iota}^{\lambda}: B(\lambda) \hookrightarrow \mathbf{Z}^{\infty} \otimes R_{\lambda}$ and described the explicit form of $\operatorname{Im} \Psi_{\iota}^{\lambda}$.

The generalized Kac-Moody algebras were introduced by Borcherds in his study of Monstrous Moonshine [1, 2]. The structure and representation theories of generalized Kac-Moody algebras are very similar to those of Kac-Moody algebras. The main difference is that the generalized Kac-Moody algebras may have imaginary simple roots with nonpositive norms whose multiplicity can be greater than one, and they may have infinitely many simple roots. In [9], Kang introduced the quantum generalized Kac-Moody algebras as an analogue of quantum groups, and in [7, Jeong, Kang, and Kashiwara developed the crystal basis theory for quantum generalized Kac-Moody algebras. In [8], Jeong, Kang, Kashiwara and the author introduced the notion of abstract crystals and an analogue $\Psi_{\iota}$ of the Kashiwara embedding of crystals for quantum generalized Kac-Moody algebras. Moreover, in [19, the author gave the generalized version of polyhedral realization of the crystal $B(\infty)$.

In this paper, we give a polyhedral realization of the crystal bases $B(\lambda)$ of the highest weight modules $V(\lambda)$ for quantum generalized Kac-Moody algebras. More precisely, we introduce the crystal $R_{\lambda}$ for generalized Kac-Moody algebras and show that the connected component containing $u_{\infty} \otimes r_{\lambda}$ is also isomorphic to the highest weight crystal $B(\lambda)$ as in the Kac-Moody case. Moreover, on the basis of the embedding of crystals given in [8] and the crystal $R_{\lambda}$ for generalized Kac-Moody algebras, we introduce the crystal structure $\mathbf{Z}_{\geq 0}^{\infty}[\lambda]$, and we give explicit images of Kashiwara embedding $\Psi_{\iota}^{\lambda}$. As applications, we give explicit descriptions of the crystals over Kac-Moody algebras of ranks 2 and 3. Moreover, for the Monster Lie algebra which played an important role in proving the Moonshine conjecture given by Conway and Norton [4, we give the explicit description of $\operatorname{Im} \Psi_{\iota}^{\lambda}$. Finally, from this description, we give characters of the highest weight module $V(\lambda)$ over $U_{q}(\mathfrak{g})$.

\section{Crystals for generalized KaC-Moody algebras}

Let $I$ be a countable index set. A real matrix $A=\left(a_{i j}\right)_{i, j \in I}$ is called a BorcherdsCartan matrix if it satisfies: (i) $a_{i i}=2$ or $a_{i i} \leq 0$ for all $i \in I$, (ii) $a_{i j} \leq 0$ if $i \neq j$, (iii) $a_{i j} \in \mathbf{Z}$ if $a_{i i}=2$, (iv) $a_{i j}=0$ if and only if $a_{j i}=0$. Let $I^{r e}=\left\{i \in I \mid a_{i i}=2\right\}$ and $I^{i m}=\left\{i \in I \mid a_{i i} \leq 0\right\}$. Moreover, we say that an index $i$ in $I^{r e}$ (resp. $I^{i m}$ ) is real (resp. imaginary).

In this paper, we assume that for all $i, j \in I, a_{i j} \in \mathbf{Z}, a_{i i} \in 2 \mathbf{Z}$, and $A$ is symmetrizable. That is, there is a diagonal matrix $D=\operatorname{diag}\left(s_{i} \in \mathbf{Z}_{>0} \mid i \in I\right)$ such that $D A$ is symmetric. We set a Borcherds-Cartan datum $\left(A, P^{\vee}, P, \Pi^{\vee}, \Pi\right)$ as follows:

$$
\begin{aligned}
& A: \text { a Borcherds-Cartan matrix, } \\
& P^{\vee}=\left(\bigoplus_{i \in I} \mathbf{Z} h_{i}\right) \oplus\left(\bigoplus_{i \in I} \mathbf{Z} d_{i}\right): \text { a free abelian group, } \\
& P=\left\{\lambda \in \mathfrak{h}^{*} \mid \lambda\left(P^{\vee}\right) \subset \mathbf{Z}\right\}: \text { the weight lattice, } \\
& \Pi^{\vee}=\left\{h_{i} \mid i \in I\right\} \subset \mathfrak{h}: \text { the set of simple coroots, } \\
& \Pi=\left\{\alpha_{i} \mid i \in I\right\} \subset \mathfrak{h}^{*}: \text { the set of simple roots. }
\end{aligned}
$$


Here, the simple roots $\alpha_{i}(i \in I)$ are defined by

$$
\left\langle h_{j}, \alpha_{i}\right\rangle=a_{j i} \text { and }\left\langle d_{j}, \alpha_{i}\right\rangle=\delta_{j i} .
$$

We denote by $U_{q}(\mathfrak{g})$ the quantum generalized Kac-Moody algebras associated with the Borcherds-Cartan datum $\left(A, P^{\vee}, P, \Pi^{\vee}, \Pi\right)$. We also denote by $P^{+}=\{\lambda \in$ $P \mid \lambda\left(h_{i}\right) \geq 0$ for all $\left.i \in I\right\}$ the set of dominant integral weights.

We recall the definition of abstract crystals introduced in [8] for quantum generalized Kac-Moody algebras.

Definition $1.1\left([\underline{8})\right.$. An abstract crystal for $U_{q}(\mathfrak{g})$ or a $U_{q}(\mathfrak{g})$-crystal is a set $B$ together with the maps wt $: B \rightarrow P, \tilde{e}_{i}, \tilde{f}_{i}: B \rightarrow B \cup\{0\}(i \in I)$, and $\varepsilon_{i}, \varphi_{i}: B \rightarrow$ $\mathbf{Z} \cup\{-\infty\}(i \in I)$ such that for all $b \in B$, we have

(i) $\operatorname{wt}\left(\tilde{e}_{i} b\right)=$ wt $b+\alpha_{i}$ if $i \in I$ and $\tilde{e}_{i} b \neq 0$,

(ii) $\operatorname{wt}\left(\tilde{f}_{i} b\right)=\mathrm{wt} b-\alpha_{i}$ if $i \in I$ and $\tilde{f}_{i} b \neq 0$,

(iii) for any $i \in I$ and $b \in B, \varphi_{i}(b)=\varepsilon_{i}(b)+\left\langle h_{i}\right.$, wt $\left.b\right\rangle$,

(iv) for any $i \in I$ and $b, b^{\prime} \in B, \tilde{f}_{i} b=b^{\prime}$ if and only if $b=\tilde{e}_{i} b^{\prime}$,

(v) for any $i \in I$ and $b \in B$ such that $\tilde{e}_{i} b \neq 0$, we have

(a) $\varepsilon_{i}\left(\tilde{e}_{i} b\right)=\varepsilon_{i}(b)-1, \varphi_{i}\left(\tilde{e}_{i} b\right)=\varphi_{i}(b)+1$ if $i \in I^{r e}$,

(b) $\varepsilon_{i}\left(\tilde{e}_{i} b\right)=\varepsilon_{i}(b)$ and $\varphi_{i}\left(\tilde{e}_{i} b\right)=\varphi_{i}(b)+a_{i i}$ if $i \in I^{i m}$,

(vi) for any $i \in I$ and $b \in B$ such that $\tilde{f}_{i} b \neq 0$, we have

(a) $\varepsilon_{i}\left(\tilde{f}_{i} b\right)=\varepsilon_{i}(b)+1$ and $\varphi_{i}\left(\tilde{f}_{i} b\right)=\varphi_{i}(b)-1$ if $i \in I^{r e}$,

(b) $\varepsilon_{i}\left(\tilde{f}_{i} b\right)=\varepsilon_{i}(b)$ and $\varphi_{i}\left(\tilde{f}_{i} b\right)=\varphi_{i}(b)-a_{i i}$ if $i \in I^{i m}$

(vii) for any $i \in I$ and $b \in B$ such that $\varphi_{i}(b)=-\infty$, we have $\tilde{e}_{i} b=\tilde{f}_{i} b=0$.

Definition 1.2 ([8]). A morphism of crystals or a crystal morphism $\psi: B_{1} \rightarrow B_{2}$ is a map $\psi: B_{1} \rightarrow B_{2}$ such that

(i) $\operatorname{wt}(\psi(b))=\operatorname{wt}(b)$ for all $b \in B_{1}$,

(ii) $\varepsilon_{i}(\psi(b))=\varepsilon_{i}(b), \varphi_{i}(\psi(b))=\varphi_{i}(b)$ for all $b \in B_{1}, i \in I$,

(iii) if $b \in B_{1}$ and $i \in I$ satisfy $\tilde{f}_{i} b \in B_{1}$, then we have $\psi\left(\tilde{f}_{i} b\right)=\tilde{f}_{i} \psi(b)$.

For a morphism of crystals $\psi: B_{1} \rightarrow B_{2}, \psi$ is called a strict morphism if

$$
\psi\left(\tilde{e}_{i} b\right)=\tilde{e}_{i} \psi(b), \psi\left(\tilde{f}_{i} b\right)=\tilde{f}_{i} \psi(b) \quad \text { for all } i \in I \text { and } b \in B_{1} .
$$

Here we understand $\psi(0)=0$. Moreover, $\psi$ is called an embedding if the underlying map $\psi: B_{1} \rightarrow B_{2}$ is injective. In this case, we say that $B_{1}$ is a subcrystal of $B_{2}$. If $\psi$ is a strict embedding, we say that $B_{1}$ is a full subcrystal of $B_{2}$.

Example 1.3. (a) The crystal basis $B(\lambda)$ of the irreducible highest weight module $V(\lambda)$ with $\lambda \in P^{+}$is a $U_{q}(\mathfrak{g})$-crystal, where the maps $\varepsilon_{i}, \varphi_{i}(i \in I)$ are given by

$$
\begin{aligned}
& \varepsilon_{i}(b)= \begin{cases}\max \left\{k \geq 0 \mid \tilde{e}_{i}^{k} b \neq 0\right\} & \text { for } i \in I^{\mathrm{re}}, \\
0 & \text { for } i \in I^{\mathrm{im}},\end{cases} \\
& \varphi_{i}(b)= \begin{cases}\max \left\{k \geq 0 \mid \tilde{f}_{i}^{k} b \neq 0\right\} & \text { for } i \in I^{\mathrm{re}}, \\
\left\langle h_{i}, \operatorname{wt}(b)\right\rangle & \text { for } i \in I^{\mathrm{im}} .\end{cases}
\end{aligned}
$$

(b) The crystal basis $B(\infty)$ of $U_{q}^{-}(\mathfrak{g})$ is a $U_{q}(\mathfrak{g})$-crystal, where

$$
\begin{aligned}
& \varepsilon_{i}(b)= \begin{cases}\max \left\{k \geq 0 \mid \tilde{e}_{i}^{k} b \neq 0\right\} & \text { for } i \in I^{\mathrm{re}}, \\
0 & \text { for } i \in I^{\mathrm{im}},\end{cases} \\
& \varphi_{i}(b)=\varepsilon_{i}(b)+\left\langle h_{i}, \operatorname{wt}(b)\right\rangle \quad(i \in I) .
\end{aligned}
$$


Example 1.4. For $\lambda \in P$, the singletons $T_{\lambda}=\left\{t_{\lambda}\right\}$ and $R_{\lambda}=\left\{r_{\lambda}\right\}$ are $U_{q}(\mathfrak{g})$ crystals with maps defined by

$$
\operatorname{wt}\left(t_{\lambda}\right)=\lambda, \quad \varepsilon_{i}\left(t_{\lambda}\right)=\varphi_{i}\left(t_{\lambda}\right)=-\infty, \quad \tilde{e}_{i} t_{\lambda}=\tilde{f}_{i} t_{\lambda}=0 \quad \text { for all } i \in I,
$$

and

$$
\operatorname{wt}\left(r_{\lambda}\right)=\lambda, \quad \varepsilon_{i}\left(r_{\lambda}\right)=-\left\langle h_{i}, \lambda\right\rangle, \quad \varphi_{i}\left(r_{\lambda}\right)=0, \quad \tilde{e}_{i} r_{\lambda}=\tilde{f}_{i} r_{\lambda}=0 \quad \text { for all } i \in I .
$$

Example 1.5. For each $i \in I$, let $B_{i}=\left\{b_{i}(-n) \mid n \geq 0\right\}$. Then $B_{i}$ is a crystal with maps defined by

$$
\begin{aligned}
& \operatorname{wt}\left(b_{i}(-n)\right)=-n \alpha_{i}, \\
& \tilde{e}_{i} b_{i}(-n)=b_{i}(-n+1), \quad \tilde{f}_{i} b_{i}(-n)=b_{i}(-n-1), \\
& \tilde{e}_{j} b_{i}(-n)=\tilde{f}_{j} b_{i}(-n)=0 \quad \text { if } j \neq i, \\
& \varepsilon_{i}\left(b_{i}(-n)\right)=n, \quad \varphi_{i}\left(b_{i}(-n)\right)=-n \quad \text { if } i \in I^{r e}, \\
& \varepsilon_{i}\left(b_{i}(-n)\right)=0, \quad \varphi_{i}\left(b_{i}(-n)\right)=-n a_{i i} \quad \text { if } i \in I^{i m}, \\
& \varepsilon_{j}\left(b_{i}(-n)\right)=\varphi_{j}\left(b_{i}(-n)\right)=-\infty \quad \text { if } j \neq i .
\end{aligned}
$$

Here, we understand $b_{i}(-n)=0$ for $n<0$. The crystal $B_{i}$ is called an elementary crystal.

We define the tensor product of a pair of crystals as follows: for two crystals $B_{1}$ and $B_{2}$, their tensor product $B_{1} \otimes B_{2}$ is $\left\{b_{1} \otimes b_{2} \mid b_{1} \in B_{1}, b_{2} \in B_{2}\right\}$ with the following crystal structure. The maps wt, $\varepsilon_{i}, \varphi_{i}$ are given by

$$
\begin{aligned}
\operatorname{wt}\left(b \otimes b^{\prime}\right) & =\operatorname{wt}(b)+\operatorname{wt}\left(b^{\prime}\right), \\
\varepsilon_{i}\left(b \otimes b^{\prime}\right) & =\max \left(\varepsilon_{i}(b), \varepsilon_{i}\left(b^{\prime}\right)-\left\langle h_{i}, \operatorname{wt}(b)\right\rangle\right), \\
\varphi_{i}\left(b \otimes b^{\prime}\right) & =\max \left(\varphi_{i}(b)+\left\langle h_{i}, \operatorname{wt}\left(b^{\prime}\right)\right\rangle, \varphi_{i}\left(b^{\prime}\right)\right) .
\end{aligned}
$$

For $i \in I$, we define

$$
\tilde{f}_{i}\left(b \otimes b^{\prime}\right)= \begin{cases}\tilde{f}_{i} b \otimes b^{\prime} & \text { if } \varphi_{i}(b)>\varepsilon_{i}\left(b^{\prime}\right), \\ b \otimes \tilde{f}_{i} b^{\prime} & \text { if } \varphi_{i}(b) \leq \varepsilon_{i}\left(b^{\prime}\right) .\end{cases}
$$

For $i \in I^{r e}$, we define

$$
\tilde{e}_{i}\left(b \otimes b^{\prime}\right)= \begin{cases}\tilde{e}_{i} b \otimes b^{\prime} & \text { if } \varphi_{i}(b) \geq \varepsilon_{i}\left(b^{\prime}\right), \\ b \otimes \tilde{e}_{i} b^{\prime} & \text { if } \varphi_{i}(b)<\varepsilon_{i}\left(b^{\prime}\right),\end{cases}
$$

and, for $i \in I^{i m}$, we define

$$
\tilde{e}_{i}\left(b \otimes b^{\prime}\right)= \begin{cases}\tilde{e}_{i} b \otimes b^{\prime} & \text { if } \varphi_{i}(b)>\varepsilon_{i}\left(b^{\prime}\right)-a_{i i}, \\ 0 & \text { if } \varepsilon_{i}\left(b^{\prime}\right)<\varphi_{i}(b) \leq \varepsilon_{i}\left(b^{\prime}\right)-a_{i i}, \\ b \otimes \tilde{e}_{i} b^{\prime} & \text { if } \varphi_{i}(b) \leq \varepsilon_{i}\left(b^{\prime}\right) .\end{cases}
$$

This tensor product rule is different from the one given in [7]. But when $B_{1}=B(\lambda)$ and $B_{2}=B(\mu)$ for $\lambda, \mu \in P^{+}$, the two rules coincide. Note that by the definition 
above, $B_{1} \otimes B_{2}$ is a crystal. Moreover, the associativity law for the tensor product holds [8].

Example 1.6. Let $R_{\lambda}=\left\{r_{\lambda}\right\}$ be the crystal given in Example 1.4. Then for a crystal $B, B \otimes R_{\lambda}$ is a crystal with the maps wt, $\varepsilon_{i}, \varphi_{i}$ given by

$$
\begin{aligned}
\operatorname{wt}\left(b \otimes r_{\lambda}\right) & =\operatorname{wt}(b)+\lambda, \\
\varepsilon_{i}\left(b \otimes r_{\lambda}\right) & =\max \left(\varepsilon_{i}(b),-\left\langle h_{i}, \lambda+\operatorname{wt}(b)\right\rangle\right), \\
\varphi_{i}\left(b \otimes r_{\lambda}\right) & = \begin{cases}\varphi_{i}(b)+\left\langle h_{i}, \lambda\right\rangle & \text { for } i \in I^{r e}, \\
\max \left(\varphi_{i}(b)+\left\langle h_{i}, \lambda\right\rangle, 0\right) & \text { for } i \in I^{i m},\end{cases} \\
\tilde{e}_{i}\left(b \otimes r_{\lambda}\right) & = \begin{cases}\tilde{e}_{i} b \otimes r_{\lambda} & \text { if } \varphi_{i}(b) \geq-\left\langle h_{i}, \lambda\right\rangle \text { and } i \in I^{r e}, \\
0 & \text { or } \varphi_{i}(b)+\left\langle h_{i}, \lambda\right\rangle+a_{i i}>0 \text { and } i \in I^{i m},\end{cases} \\
\tilde{f}_{i}\left(b \otimes r_{\lambda}\right) & = \begin{cases}\tilde{f}_{i} b \otimes r_{\lambda} & \text { if } \varphi_{i}(b)>-\left\langle h_{i}, \lambda\right\rangle, \\
0 & \text { otherwise. }\end{cases}
\end{aligned}
$$

\section{Embedding of the highest Weight CRystal $B(\lambda)$}

Let $*$ be the $\mathbf{Q}(q)$-algebra anti-automorphism of $U_{q}(\mathfrak{g})$ such that

$$
e_{i}^{*}=e_{i}, f_{i}^{*}=f_{i} \text { and }\left(q^{h}\right)^{*}=q^{-h} .
$$

In $\left[8\right.$, it is verified that $L(\infty)^{*}=L(\infty)$ and $B(\infty)^{*}=B(\infty)$. So let us define for each $i \in I^{r e}, \varepsilon_{i}^{*}(b)=\varepsilon_{i}\left(b^{*}\right)$ and $\varphi_{i}^{*}(b)=\varphi\left(b^{*}\right)$.

Proposition $2.1([8])$. For all $i \in I$, there exists a unique strict embedding

$$
\Psi_{i}: B(\infty) \longrightarrow B(\infty) \otimes B_{i} \quad \text { such that } u_{\infty} \mapsto u_{\infty} \otimes b_{i}(0),
$$

where $u_{\infty}$ is the highest weight vector in $B(\infty)$.

Proof. Now, we give a brief sketch of the proof given in [8]. For $b=\tilde{f}_{i_{r}} \ldots \tilde{f}_{i_{1}} u_{\infty} \in$ $B(\infty)$, take $\mu \gg 0$ such that $\widehat{\pi}_{\mu}(b)=\widehat{\pi}_{\mu}\left(\tilde{f}_{i_{r}} \ldots \tilde{f}_{i_{1}} u_{\infty}\right)=\tilde{f}_{i_{r}} \ldots \tilde{f}_{i_{1}} u_{\mu} \neq 0$. Let $\lambda \in P^{+}$such that $\lambda\left(h_{i}\right)=0$ and $\lambda\left(h_{j}\right)=\mu\left(h_{j}\right) \gg 0$ for all $j \neq i$. Set $\mu\left(h_{i}\right)=l \gg 0$. Then there is a strict embedding $\phi: B(\mu) \rightarrow B(\lambda) \otimes B\left(l \Lambda_{i}\right)$ sending $u_{\mu}$ to $u_{\lambda} \otimes u_{l \Lambda_{i}}$. Moreover, $\operatorname{Im}\left(\phi \circ \widehat{\pi}_{\mu}\right)$ belongs to $B(\lambda) \otimes\left\{\tilde{f}_{i}^{m} u_{l \Lambda_{i}} \mid m \geq 0\right\}$.

Define a map $\psi_{i}: B(\lambda) \otimes\left\{\tilde{f}_{i}^{m} u_{l \Lambda_{i}} \mid m \geq 0\right\} \rightarrow B(\infty) \otimes B_{i}$ by

$$
\psi_{i}\left(\tilde{f}_{j_{s}} \ldots \tilde{f}_{j_{1}} u_{\lambda} \otimes \tilde{f}_{i}^{m} u_{l \Lambda_{i}}\right)=\tilde{f}_{j_{s}} \ldots \tilde{f}_{j_{1}} u_{\infty} \otimes b_{i}(-m) .
$$

Then $\psi_{i}$ is injective, and $\psi_{i}$ commutes with $\tilde{e}_{j}$ and $\tilde{f}_{j}$.

Now, we define $\Psi_{i}(b)$ by

$$
\begin{aligned}
\psi_{i} \circ \phi \circ \widehat{\pi}_{\mu}(b) & =\psi_{i} \circ \phi\left(\tilde{f}_{i_{r}} \ldots \tilde{f}_{i_{1}} u_{\mu}\right)=\psi_{i}\left(\tilde{f}_{i_{r}} \ldots \tilde{f}_{i_{1}}\left(u_{\lambda} \otimes u_{l \Lambda_{i}}\right)\right) \\
& =\psi_{i}\left(\tilde{f}_{j_{s}} \ldots \tilde{f}_{j_{1}}\left(u_{\lambda} \otimes \tilde{f}_{i}^{m} u_{l \Lambda_{i}}\right)\right)=\tilde{f}_{j_{s}} \ldots \tilde{f}_{j_{1}} u_{\infty} \otimes b_{i}(-m) .
\end{aligned}
$$

Then $\Psi_{i}$ is a strict embedding. Moreover,

$$
\tilde{f}_{j_{s}} \ldots \tilde{f}_{j_{1}} u_{\infty}=\left(\tilde{e}_{i}^{m} b^{*}\right)^{*}, \text { and } m=\max _{k}\left\{k \mid \tilde{e}_{i}^{k} b^{*} \neq 0\right\} .
$$


Let $\iota$ be an infinite sequence, $\iota=\left(\ldots, i_{2}, i_{1}\right)$ in $I$, such that every $i \in I$ appears infinitely many times, and let $\mathbf{Z}_{\geq 0, \iota}^{\infty}$ be the crystal introduced in [19. (Indeed, the crystal $\mathbf{Z}_{\geq 0, \iota}^{\infty}$ is isomorphic to the crystal $B(\iota)$, the (set-theoretical) inductive limit of $B(k)=B_{i_{k}} \otimes \cdots \otimes B_{i_{1}}$, given in [8].) By taking the composition of the above crystal embeddings repeatedly, we have

Proposition 2.2 ([8, 19]). There is a strict embedding $\Psi_{\iota}: B(\infty) \hookrightarrow \mathbf{Z}_{\geq 0, \iota}^{\infty}$.

Let $\pi_{\lambda}: U_{q}^{-}(\mathfrak{g}) \rightarrow V(\lambda)$ be the natural projection sending $L(\infty)$ to $L(\lambda)$, and let $\widehat{\pi}_{\lambda}: L(\infty) / q L(\infty) \rightarrow L(\lambda) / q L(\lambda)$ be the induced map sending $B(\infty)$ to $B(\lambda) \cup\{0\}$. Then we have

$$
\begin{gathered}
\tilde{f}_{i} \circ \widehat{\pi}_{\lambda}=\widehat{\pi}_{\lambda} \circ \tilde{f}_{i}, \\
\tilde{e}_{i} \circ \widehat{\pi}_{\lambda}=\widehat{\pi}_{\lambda} \circ \tilde{e}_{i}, \quad \text { if } \widehat{\pi}_{\lambda}(b) \neq 0, \\
\widehat{\pi}_{\lambda}: B(\infty) \backslash\left\{\widehat{\pi}_{\lambda}^{-1}(0)\right\} \rightarrow B(\lambda) \text { is bijective. }
\end{gathered}
$$

Now, define a map

$$
\Phi_{\lambda}:\left(B(\infty) \otimes R_{\lambda}\right) \cup\{0\} \rightarrow B(\lambda) \cup\{0\}
$$

by

$$
\Phi_{\lambda}\left(b \otimes r_{\lambda}\right)=\widehat{\pi}_{\lambda}(b) \quad \text { and } \quad \Phi_{\lambda}(0)=0 .
$$

Let $\widetilde{B}(\lambda):=\left\{b \otimes r_{\lambda} \in B(\infty) \otimes R_{\lambda} \mid \Phi_{\lambda}\left(b \otimes r_{\lambda}\right) \neq 0\right\}$. Then we have

Theorem 2.3. (a) The map $\Phi_{\lambda}$ is a surjective strict morphism of crystals. Moreover, it induces an isomorphism of crystals from $\widetilde{B}(\lambda)$ to $B(\lambda)$.

(b) $\widetilde{B}(\lambda)$ is the set of $b \otimes r_{\lambda} \in B(\infty) \otimes R_{\lambda}$ such that

$$
\text { (i) } \varepsilon_{i}^{*}(b) \leq \lambda\left(h_{i}\right)\left(i \in I^{r e}\right), \quad \text { (ii) if } \lambda\left(h_{i}\right)=0, \tilde{e}_{i} b^{*}=0 .
$$

Proof. (b) is directly derived from the following fact, obtained by (2.1) in the proof of Proposition 2.1.

$$
\begin{gathered}
\widehat{\pi}_{\lambda}(b) \neq 0 \text { if and only if } \\
\varepsilon_{i}^{*}(b) \leq \lambda\left(h_{i}\right)\left(i \in I^{r e}\right), \text { and } \lambda\left(h_{i}\right)=0 \text { implies } \tilde{e}_{i} b^{*}=0 .
\end{gathered}
$$

For (a), since $\widehat{\pi}_{\lambda}$ is surjective, it is clear that $\Phi_{\lambda}$ is also surjective. In order to show that $\Phi_{\lambda}$ is a strict morphism of crystals, it suffices to show that for $u \in$ $B(\infty) \otimes R_{\lambda}$,

(1) $\operatorname{wt}\left(\Phi_{\lambda}(u)\right)=\operatorname{wt}(u)$ if $\Phi_{\lambda}(u) \neq 0$,

(2) $\varepsilon_{i}\left(\Phi_{\lambda}(u)\right)=\varepsilon_{i}(u)$ for any $i \in I$ if $\Phi_{\lambda}(u) \neq 0$,

(3) $\varphi_{i}\left(\Phi_{\lambda}(u)\right)=\varphi_{i}(u)$ for any $i \in I$ if $\Phi_{\lambda}(u) \neq 0$,

(4) $\tilde{e}_{i} \Phi_{\lambda}(u)=\Phi_{\lambda}\left(\tilde{e}_{i} u\right)$ for any $i$,

(5) $\tilde{f}_{i} \Phi_{\lambda}(u)=\Phi_{\lambda}\left(\tilde{f}_{i} u\right)$ for any $i$.

If $i \in I^{r e},(1),(2)$ and (3) follow from the proof of Theorem 3.1 of [17, and if $i \in I^{i m}$, since $\varepsilon_{i}(b)=0$ for all $b \in B(\infty)$ or $B(\lambda)$, it is clear. So it suffices to consider (4) and (5). For $u=b \otimes r_{\lambda}$, suppose that $\Phi_{\lambda}(u)=\widehat{\pi}_{\lambda}(b) \neq 0$. When $i \in I^{r e}$, we have

$$
0 \leq \varphi_{i}\left(\widehat{\pi}_{\lambda}(b)\right)=\left\langle h_{i}, \lambda\right\rangle+\varphi_{i}(b),
$$

which implies that $\tilde{e}_{i}\left(b \otimes r_{\lambda}\right)=\tilde{e}_{i} b \otimes r_{\lambda}$. Hence

$$
\tilde{e}_{i} \Phi_{\lambda}(u)=\tilde{e}_{i} \widehat{\pi}_{\lambda}(b)=\widehat{\pi}_{\lambda}\left(\tilde{e}_{i} b\right)=\Phi_{\lambda}\left(\tilde{e}_{i} u\right) .
$$


When $i \in I^{i m}$, if $\tilde{e}_{i}\left(b \otimes r_{\lambda}\right)=\tilde{e}_{i} b \otimes r_{\lambda}$, there is nothing to prove. If $\tilde{e}_{i}\left(b \otimes r_{\lambda}\right)=$ $b \otimes \tilde{e}_{i} r_{\lambda}=0$, then $\left\langle h_{i}, \lambda+\operatorname{wt}(b)\right\rangle+a_{i i} \leq 0$. Hence $\tilde{e}_{i} \Phi_{\lambda}(u)=\tilde{e}_{i} \widehat{\pi}_{\lambda}(b)=0=\Phi_{\lambda}\left(\tilde{e}_{i} u\right)$.

Next, consider the case $\Phi_{\lambda}(u)=\widehat{\pi}_{\lambda}(b)=0$. Then it suffices to show that $\Phi_{\lambda}\left(\tilde{e}_{i}\left(b \otimes r_{\lambda}\right)\right)=0$. If $\tilde{e}_{i}\left(b \otimes r_{\lambda}\right)=0$, there is nothing to prove. If $\tilde{e}_{i}\left(b \otimes r_{\lambda}\right) \neq 0$, since $\tilde{e}_{i} r_{\lambda}=0$,

$$
\tilde{e}_{i}\left(b \otimes r_{\lambda}\right)=\tilde{e}_{i} b \otimes r_{\lambda}
$$

which implies

$$
\begin{aligned}
& \varphi_{i}(b) \geq \varepsilon_{i}\left(r_{\lambda}\right)=-\left\langle h_{i}, \lambda\right\rangle \quad \text { for } i \in I^{r e} \\
& \left\langle h_{i}, \operatorname{wt}(b)+\lambda\right\rangle+a_{i i}>0 \quad \text { for } i \in I^{i m} .
\end{aligned}
$$

Assuming $\Phi_{\lambda}\left(\tilde{e}_{i} b \otimes r_{\lambda}\right)=\widehat{\pi}_{\lambda}\left(\tilde{e}_{i} b\right) \neq 0$, we shall derive a contradiction. Since $\tilde{f}_{i}$ and $\widehat{\pi}_{\lambda}$ commute, $\tilde{f}_{i} \widehat{\pi}_{\lambda}\left(\tilde{e}_{i} b\right)=\widehat{\pi}_{\lambda}\left(\tilde{f}_{i} \tilde{e}_{i} b\right)=\widehat{\pi}_{\lambda}(b)=0$. It means that

$$
\varphi_{i}\left(\widehat{\pi}_{\lambda}\left(\tilde{e}_{i} b\right)\right)=0 \quad\left(i \in I^{r e}\right) \quad \text { and } \quad\left\langle h_{i}, \widehat{\pi}_{\lambda}\left(\tilde{e}_{i} b\right)\right\rangle=0 \quad\left(i \in I^{i m}\right) .
$$

When $i \in I^{r e}$,

$$
\begin{aligned}
0 & =\varphi_{i}\left(\widehat{\pi}_{\lambda}\left(\tilde{e}_{i} b\right)\right)=\left\langle h_{i}, \operatorname{wt}\left(\widehat{\pi}_{\lambda}\left(\tilde{e}_{i} b\right)\right)\right\rangle+\varepsilon_{i}\left(\widehat{\pi}_{\lambda}\left(\tilde{e}_{i} b\right)\right) \\
& =\left\langle h_{i}, \lambda+\operatorname{wt}(b)+\alpha_{i}\right\rangle+\varepsilon_{i}\left(\tilde{e}_{i} b\right) \\
& =\left\langle h_{i}, \lambda\right\rangle+\left\langle h_{i}, \operatorname{wt}(b)\right\rangle+2+\varepsilon_{i}(b)-1 \\
& =\left\langle h_{i}, \lambda\right\rangle+\varphi_{i}(b)+1 .
\end{aligned}
$$

Therefore,

$$
\varphi_{i}(b)=-\left\langle h_{i}, \lambda\right\rangle-1=\varepsilon_{i}\left(r_{\lambda}\right)-1<\varepsilon_{i}\left(r_{\lambda}\right),
$$

which contradicts (2.3).

When $i \in I^{i m}$,

$$
0=\left\langle h_{i}, \widehat{\pi}_{\lambda}\left(\tilde{e}_{i} b\right)\right\rangle=\left\langle h_{i}, \operatorname{wt}(b)+\lambda+\alpha_{i}\right\rangle .
$$

This contradicts (2.3).

Now, let us prove (5). We know that $\tilde{f}_{i} \Phi_{\lambda}(u)=\tilde{f}_{i} \widehat{\pi}_{\lambda}(b)=\widehat{\pi}_{\lambda}\left(\tilde{f}_{i} b\right)$. So if $\tilde{f}_{i} u=\tilde{f}_{i} b \otimes r_{\lambda}$, then $\widehat{\pi}_{\lambda}\left(\tilde{f}_{i} b\right)=\Phi_{\lambda}\left(\tilde{f}_{i} u\right)$. Suppose that $\tilde{f}_{i} u=b \otimes \tilde{f}_{i} r_{\lambda}=0$. Then we have

$$
\begin{aligned}
& \varphi_{i}(b) \leq \varepsilon_{i}\left(r_{\lambda}\right)=-\left\langle h_{i}, \lambda\right\rangle \quad \text { for } i \in I^{r e}, \\
& \left\langle h_{i}, \operatorname{wt}(b)+\lambda\right\rangle=0 \quad \text { for } i \in I^{i m} .
\end{aligned}
$$

If $\Phi_{\lambda}(u)=\widehat{\pi}_{\lambda}(b)=0$, there is nothing to prove. Assume that $\Phi_{\lambda}(u)=\widehat{\pi}_{\lambda}(b) \neq 0$. Suppose that $\tilde{f}_{i} \Phi_{\lambda}(u)=\tilde{f}_{i} \widehat{\pi}_{\lambda}(b) \neq 0$. Then

$$
\begin{aligned}
& 0<\varphi_{i}\left(\widehat{\pi}_{\lambda}(b)\right)=\left\langle h_{i}, \lambda\right\rangle+\varphi_{i}(b) \text { for } i \in I^{r e}, \\
& \left\langle h_{i}, \lambda+\operatorname{wt}(b)\right\rangle>0 \text { for } i \in I^{i m},
\end{aligned}
$$

which contradicts (2.4). Therefore, $\Phi_{\lambda}$ is a surjective strict morphism of crystals.

Finally, we will show that $\Phi_{\lambda}$ induces an isomorphism of crystals from $\widetilde{B}(\lambda)$ to $B(\lambda)$. Since $\Phi_{\lambda}\left(b \otimes r_{\lambda}\right) \neq 0$ is equivalent to $\widehat{\pi}_{\lambda}(b) \neq 0, \phi_{\lambda}:=\left.\Phi_{\lambda}\right|_{\widetilde{B}(\lambda)}: \widetilde{B}(\lambda) \rightarrow B(\lambda)$ is a 1-1 correspondence. So if we show that $\widetilde{B}(\lambda)$ is stable under $\tilde{e}_{i}$ and $\tilde{f}_{i}$, then $\phi_{\lambda}$ is a strict morphism of crystals. In order to prove stability, it is enough to show that $\Phi_{\lambda}\left(\tilde{e}_{i}\left(b \otimes r_{\lambda}\right)\right)=0$ (resp. $\left.\Phi_{\lambda}\left(\tilde{f}_{i}\left(b \otimes r_{\lambda}\right)\right)=0\right)$ implies $\tilde{e}_{i}\left(b \otimes r_{\lambda}\right)=0$ (resp. $\left.\tilde{f}_{i}\left(b \otimes r_{\lambda}\right)=0\right)$. First, suppose that $\Phi_{\lambda}\left(\tilde{e}_{i}\left(b \otimes r_{\lambda}\right)\right)=\tilde{e}_{i} \widehat{\pi}_{\lambda}(b)=0$. Since $\widehat{\pi}_{\lambda}(b) \neq 0$, $\tilde{e}_{i} \widehat{\pi}_{\lambda}(b)=\widehat{\pi}_{\lambda}\left(\tilde{e}_{i} b\right)=0$, which implies $\tilde{e}_{i} b=0$. Indeed, if $\tilde{e}_{i} b \neq 0$, we have

$$
\max _{k}\left\{k \mid \tilde{e}_{j}^{k}\left(\tilde{e}_{i} b\right)^{*} \neq 0\right\} \leq \max _{k}\left\{k \mid \tilde{e}_{j}^{k} b^{*} \neq 0\right\} \quad \text { for any } j \in I \text {. }
$$


This contradicts $\widehat{\pi}_{\lambda}\left(\tilde{e}_{i} b\right)=0$ by (2.2). Therefore, we have $\tilde{e}_{i}\left(b \otimes r_{\lambda}\right)=0$. Secondly, suppose that $\Phi_{\lambda}\left(\tilde{f}_{i}\left(b \otimes r_{\lambda}\right)\right)=\tilde{f}_{i} \widehat{\pi}_{\lambda}(b)=0$. If $i \in I^{r e}$, we have $0=\varphi_{i}\left(\widehat{\pi}_{\lambda}(b)\right)=$ $\varphi_{i}(b)+\left\langle h_{i}, \lambda\right\rangle$. Therefore, $\tilde{f}_{i}\left(b \otimes r_{\lambda}\right)=b \otimes \tilde{f}_{i} r_{\lambda}=0$. If $i \in I^{i m}$, we have $0=$ $\left\langle h_{i}, \operatorname{wt}\left(\widehat{\pi}_{\lambda}(b)\right)\right\rangle=\left\langle h_{i}, \lambda+\operatorname{wt}(b)\right\rangle$. Therefore, $\tilde{f}_{i}\left(b \otimes r_{\lambda}\right)=0$.

Let $\iota=\left(\ldots, i_{k}, \ldots, i_{1}\right)$ be an infinite sequence such that

$$
i_{k} \neq i_{k+1} \quad \text { and } \quad \#\left\{k: i_{k}=i\right\}=\infty \quad \text { for any } i \in I .
$$

According to Proposition 2.2 and Theorem 2.3, we derive a crystal structure $\mathbf{Z}_{\geq 0, \iota}^{\infty}[\lambda]$ on the set of infinite sequences of nonnegative integers

$$
\mathbf{Z}_{\geq 0}^{\infty}:=\left\{\left(\ldots, x_{k}, \ldots, x_{1}\right): x_{k} \in \mathbf{Z}_{\geq 0} \text { and } x_{k}=0 \text { for } k \gg 0\right\}
$$

associated with $\iota$ as follows: Let $\vec{x}=\left(\ldots, x_{k}, \ldots, x_{1}\right)$ be an element of $\mathbf{Z}_{\geq 0}^{\infty}$ corresponding to $\cdots \otimes b_{i_{k}}\left(-x_{k}\right) \otimes \cdots \otimes b_{i_{1}}\left(-x_{1}\right) \otimes r_{\lambda}$. For $k \geq 1$, we define

$$
\sigma_{k}(\vec{x})= \begin{cases}x_{k}+\sum_{j>k}\left\langle h_{i_{k}}, \alpha_{i_{j}}\right\rangle x_{j} & \text { if } i_{k} \in I^{r e}, \\ \sum_{j>k}\left\langle h_{i_{k}}, \alpha_{i_{j}}\right\rangle x_{j} & \text { if } i_{k} \in I^{i m} .\end{cases}
$$

Let

$$
\begin{aligned}
& \sigma^{(i)}(\vec{x})=\max _{k: i_{k}=i}\left\{\sigma_{k}(\vec{x})\right\}, \\
& \sigma_{0}^{(i)}(\vec{x})=-\left\langle h_{i}, \lambda\right\rangle+\sum_{j \geq 1}\left\langle h_{i}, \alpha_{i_{j}}\right\rangle x_{j}, \\
& n_{f}=\min \left\{k: i_{k}=i, \sigma_{k}(\vec{x})=\sigma^{(i)}(\vec{x})\right\}, \\
& n_{e}= \begin{cases}\max \left\{k: i_{k}=i, \sigma_{k}(\vec{x})=\sigma^{(i)}(\vec{x})\right\} & \text { if } i \in I^{r e}, \\
n_{f} & \text { if } i \in I^{i m} .\end{cases}
\end{aligned}
$$

For each $k \geq 1$, we denote by $k^{(+)}$(resp. $k^{(-)}$) the minimal (resp. maximal) index $j>k$ (resp. $j<k$ ) such that $i_{j}=i_{k}$. We now define

$$
\tilde{f}_{i} \vec{x}= \begin{cases}\left(x_{k}+\delta_{k, n_{f}}: k \geq 1\right) & \text { if } i \in I^{r e} \text { and } \sigma^{(i)}(\vec{x})>\sigma_{0}^{(i)}(\vec{x}), \\ & \text { or } i \in I^{i m} \text { and } \sigma_{0}^{(i)}(\vec{x})<0, \\ & \text { otherwise }\end{cases}
$$

and $\tilde{e}_{i} \vec{x}=\left(x_{k}-\delta_{k, n_{e}}: k \geq 1\right)$ if $\vec{x}$ satisfies the following conditions:

$$
\begin{aligned}
& \text { (i) } i \in I^{r e}: \sigma^{(i)}(\vec{x})>0, \sigma^{(i)}(\vec{x}) \geq \sigma_{0}^{(i)}(\vec{x}), \\
& \text { (ii) } i \in I^{i m}: \sigma_{0}^{(i)}(\vec{x})-a_{i i}<0 \text {, for } k=n_{e} \text { with } k^{(-)} \neq 0, \\
& x_{k}>1 \text {, or } x_{k}=1 \text { and } \sum_{k^{(-)}<j<k}\left\langle h_{i}, \alpha_{i_{j}}\right\rangle x_{j}<0 .
\end{aligned}
$$

Otherwise, $\tilde{e}_{i} \vec{x}=0$. We also define

$$
\begin{aligned}
& \operatorname{wt}(\vec{x})=\lambda-\sum_{j=1}^{\infty} x_{j} \alpha_{i_{j}}, \\
& \varepsilon_{i}(\vec{x})=\max \left(\sigma^{(i)}(\vec{x}), \sigma_{0}^{(i)}(\vec{x})\right), \\
& \varphi_{i}(\vec{x})=\left\langle h_{i}, \operatorname{wt}(\vec{x})\right\rangle+\varepsilon_{i}(\vec{x}) .
\end{aligned}
$$


Then it is easy to see that $\mathbf{Z}_{\geq 0}^{\infty}$ is a crystal, and we will denote this crystal by $\mathbf{Z}_{\geq 0, \iota}^{\infty}[\lambda]$. Note that the crystal $\mathbf{Z}_{\geq 0, \iota}^{\infty}[\lambda]$ is isomorphic to $\mathbf{Z}_{\geq 0, \iota}^{\infty} \otimes R_{\lambda} \cong B(\iota) \otimes R_{\lambda}$. Therefore, by Theorem 2.3, we have

Proposition 2.4. Let $B(\lambda)$ be the highest weight crystal with a dominant integral weight. Then there is a strict embedding $\Psi_{\iota}^{\lambda}: B(\lambda) \hookrightarrow \mathbf{Z}_{\geq 0, \iota}^{\infty}[\lambda]$.

\section{Polyhedral Realization of $B(\lambda)$}

Let $\mathbf{Q}^{\infty}=\left\{\vec{x}=\left(\ldots, x_{k}, \ldots, x_{1}\right) \mid x_{k} \in \mathbf{Q}\right.$ and $x_{k}=0$ for $\left.k \gg 0\right\}$ be an infinite dimensional vector space. Let $\beta_{k}^{( \pm)}(\vec{x})$ be linear functions given by

$$
\beta_{k}^{(+)}(\vec{x})=\sigma_{k}(\vec{x})-\sigma_{k(+)}(\vec{x})
$$

and

$$
\beta_{k}^{(-)}(\vec{x})= \begin{cases}\sigma_{k^{(-)}}(\vec{x})-\sigma_{k}(\vec{x}) & \text { if } k^{(-)}>0, \\ \sigma_{0}^{\left(i_{k}\right)}(\vec{x})-\sigma_{k}(\vec{x}) & \text { if } k^{(-)}=0 .\end{cases}
$$

We define an operator $\widehat{S}_{k}=\widehat{S}_{k, \iota}$ for a linear function $\psi(\vec{x})=c+\sum_{k \geq 1} \psi_{k} x_{k}$ on $\left(\mathbf{Q}^{\infty}\right)^{*}$ by

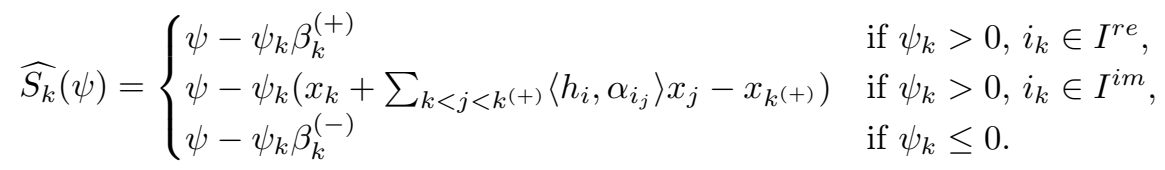

For the fixed sequence $\iota$, we denote by $\iota^{(i)}$ the first number $k$ such that $i_{k}=i$. For each $i \in I^{r e}$, let

$$
\lambda^{(i)}(\vec{x})=\left\langle h_{i}, \lambda\right\rangle-\sum_{1 \leq j<\iota(i)}\left\langle h_{i}, \alpha_{i_{j}}\right\rangle x_{j}-x_{\iota^{(i)}} .
$$

For $\iota$ and a dominant integral weight $\lambda$,

$$
\begin{aligned}
\Theta_{\iota}[\lambda]:= & \left\{\widehat{S}_{j_{l}} \cdots \widehat{S}_{j_{1}} x_{j_{0}}: l \geq 0, j_{0}, \ldots, j_{l} \geq 1\right\} \\
& \cup\left\{\widehat{S}_{j_{k}} \cdots \widehat{S}_{j_{1}} \lambda^{(i)}(\vec{x}): k \geq 0, j_{1}, \ldots, j_{k} \geq 1\right\} .
\end{aligned}
$$

Moreover, for a given $s, t \geq 1(t>s)$, let $\Theta_{\iota}^{s \backslash t}$ be the subset of $\Theta_{\iota}$ of linear forms obtained from the coordinate forms $x_{s}$ by applying transformations $S_{k}$ with $k \neq t$, i.e.,

$$
\Theta_{\iota}^{s \backslash t}[\lambda]=\left\{\widehat{S}_{j_{l}} \cdots \widehat{S}_{j_{1}} x_{s}: l \geq 0, s, j_{1}, \ldots, j_{l} \geq 1\right\},
$$

where none of $j_{1}, \ldots, j_{l}$ is $t$.

Let $\Gamma_{\iota}[\lambda]$ be the set of $\vec{x} \in \mathbf{Z}_{\geq 0, \iota}^{\infty}[\lambda]$ satisfying the following conditions:

(i) $\psi(\vec{x}) \geq 0$ for any $\psi \in \Theta_{\iota}[\lambda]$,

(ii) for each $t$ with $i_{t} \in I^{i m}$, if $x_{t} \neq 0$, then

$$
\begin{aligned}
\sum_{t^{(-)}<j<t}\left\langle h_{i_{t}}, \alpha_{i_{j}}\right\rangle x_{j}<0 & \left(t^{(-)} \neq 0\right), \\
-\left\langle h_{i_{t}}, \lambda\right\rangle+\sum_{1 \leq j<t}\left\langle h_{i_{t}}, \alpha_{i_{j}}\right\rangle x_{j}<0 & \left(t^{(-)}=0\right) .
\end{aligned}
$$


In addition, if

$$
\begin{aligned}
& \sum_{\substack{t(-)<j<t \\
j \in I^{i m}}}\left\langle h_{i_{t}}, \alpha_{i_{j}}\right\rangle x_{j}=0\left(t^{(-)} \neq 0\right), \\
&-\left\langle h_{i_{t}}, \lambda\right\rangle+\sum_{\substack{1 \leq j<t \\
j \in I^{i m}}}\left\langle h_{i_{t}}, \alpha_{i_{j}}\right\rangle x_{j}=0 \quad\left(t^{(-)}=0\right),
\end{aligned}
$$

then there exists a $p\left(t^{(-)}<p<t\right)$ with $i_{p} \in I^{r e}$ such that

$$
\left\langle h_{i_{t}}, \alpha_{i_{p}}\right\rangle x_{p}<0 \text { and } \psi(\vec{x})>0 \text { for any } \psi \in \Theta_{\iota}^{p \backslash t}[\lambda] .
$$

Theorem 3.1. Let $\iota$ be the sequence of indices satisfying (2.5), and let $\lambda$ be a dominant integral weight. Suppose that $\overrightarrow{0}=(\ldots, 0, \ldots, 0)$ belongs to $\Gamma_{\iota}[\lambda]$. Let $\Psi_{\iota}^{\lambda}: B(\lambda) \hookrightarrow \mathbf{Z}_{\geq 0, \iota}^{\infty}[\lambda]$ be the embedding. Then we have

$$
\operatorname{Im} \Psi_{\iota}^{\lambda}(\cong \widetilde{B}(\lambda))=\Gamma_{\iota}[\lambda] .
$$

Proof. First, let us start by showing that $\Gamma_{\iota}[\lambda]$ is closed under the Kashiwara operators $\tilde{e}_{i}$ and $\tilde{f}_{i}$. Let $\vec{x}$ be an element of $\Gamma_{\iota}[\lambda]$. Suppose that $\tilde{f}_{i} \vec{x}=$ $\left(\ldots, x_{k}+1, \ldots, x_{2}, x_{1}\right) \neq 0$. Since $\psi\left(\tilde{f}_{i} \vec{x}\right)=\psi(\vec{x})+\psi_{k} \geq \psi_{k}$ for any $\psi \in \Gamma_{\iota}[\lambda]$, it suffices to consider the case $\psi_{k}<0$. Note that for each $k, \sigma_{k}(\vec{x})>\sigma_{k^{(-)}}(\vec{x})$ if $k^{(-)}>0$, and $\sigma_{k}(\vec{x})>\sigma_{0}^{(i)}(\vec{x})$ if $k^{(-)}=0$. (Indeed, when $i_{k} \in I^{i m}, \sigma_{k}(\vec{x})=0$, and $\sigma_{k^{(-)}}(\vec{x})<0$ and $\sigma_{0}^{(i)}(\vec{x})<0$.) So we have $\beta_{k}^{(-)}(\vec{x}) \leq-1$, which implies

$$
\psi\left(\tilde{f}_{i} \vec{x}\right)=\psi(\vec{x})+\psi_{k} \geq \psi(\vec{x})-\psi_{k} \beta_{k}^{(-)}(\vec{x})=\left(\widehat{S_{k}} \psi\right)(\vec{x}) \geq 0 .
$$

Now, suppose that $\tilde{f}_{i} \vec{x}$ does not satisfy the condition (3.3). Then we have the following cases:

(i) $k^{(-)} \neq 0: \quad x_{k}=0, \sum_{k^{(-)}<j<k}\left\langle h_{i}, \alpha_{i_{j}}\right\rangle x_{j}=0 \quad$ (in $\left.\vec{x}\right)$

$$
\begin{aligned}
\text { (ii) } k^{(-)}=0: & \left.\stackrel{\tilde{f}_{i}}{\longrightarrow} \quad x_{k}=1, \sum_{k(-)<j<k}\left\langle h_{i}, \alpha_{i_{j}}\right\rangle x_{j}=0 \quad \text { (in } \tilde{f}_{i} \vec{x}\right), \\
& \left.x_{k}=0,-\left\langle h_{i}, \lambda\right\rangle+\sum_{1 \leq j<k}\left\langle h_{i}, \alpha_{i_{j}}\right\rangle x_{j}=0 \quad \text { (in } \vec{x}\right) \\
& \stackrel{\tilde{f}_{i}}{\longrightarrow} \quad x_{k}=1,-\left\langle h_{i}, \lambda\right\rangle+\sum_{1 \leq j<k}\left\langle h_{i}, \alpha_{i_{j}}\right\rangle x_{j}=0 \quad\left(\text { in } \tilde{f}_{i} \vec{x}\right) .
\end{aligned}
$$

But, this cannot occur by the definition of the Kashiwara operator $\tilde{f}_{i}$.

Now, we show that $\tilde{f}_{i} \vec{x}$ satisfies the condition (3.5). First, suppose that there exist $p$ and $t$ satisfying (3.5) in $\vec{x}$. Since $\psi\left(\tilde{f}_{i} \vec{x}\right)=\psi(\vec{x})+\psi_{k}$, it is enough to consider the cases that $\psi_{k}<0$. Note that by definition of the set $\Theta_{\iota}^{p \backslash t}[\lambda]$, $\psi_{t}>0$ for all $\psi \in \Theta_{\iota}^{p \backslash t}[\lambda]$. So it suffices to consider the case that $k \neq t$. If $k \neq t$, then $S_{k} \psi \in \Theta_{\iota}^{p \backslash t}$ and so $\psi\left(\tilde{f}_{i} \vec{x}\right)=\psi(\vec{x})+\psi_{k} \geq\left(S_{k} \psi\right)(\vec{x})>0$. Second, suppose that $k=t, x_{t}=0$, and for any $j$ such that $t^{(-)}<j<t, i_{j} \in I^{r e}$, $\left\langle h_{i_{t}}, \alpha_{i_{j}}\right\rangle x_{j}<0$, there is a $\psi \in \Theta_{\iota}^{j \backslash t}[\lambda]$ such that $\psi(\vec{x})=0$ in $\vec{x}$. Note that since $j$ is the index such that $\left\langle h_{i_{t}}, \alpha_{i_{j}}\right\rangle<0$, we have $\psi_{t}>0$ for all $\psi \in \Theta_{\iota}^{j \backslash t}[\lambda]$. Therefore, $\psi\left(\tilde{f}_{i} \vec{x}\right)=\psi(\vec{x})+\psi_{t} \geq \psi_{t}>0$ for all $\psi \in \Theta_{\iota}^{j \backslash t}[\lambda]$. Therefore, $\Gamma_{\iota}[\lambda]$ is closed under $\tilde{f}_{i}$.

On the other hand, suppose that $\tilde{e}_{i} \vec{x}=\left(\ldots, x_{k}-1, \ldots, x_{2}, x_{1}\right) \neq 0$. Since $\psi\left(\tilde{e}_{i} \vec{x}\right)=\psi(\vec{x})-\psi_{k} \geq-\psi_{k}$, it is enough to consider the case $\psi_{k}>0$. Note that if $i_{k} \in I^{r e}, \sigma_{k}(\vec{x})>\sigma_{k^{(+)}}(\vec{x})$, and if $i_{k} \in I^{i m}$, then $x_{k}+\sum_{k<j<k^{(+)}}\left\langle h_{i_{k}}, \alpha_{i_{j}}\right\rangle x_{j}-$ 
$x_{k^{(+)}}>0$. Therefore,

$$
\begin{aligned}
& \psi\left(\tilde{e}_{i} \vec{x}\right)=\psi(\vec{x})-\psi_{k} \\
& \geq \begin{cases}\psi(\vec{x})-\psi_{k} \beta_{k}^{(+)}(\vec{x}) & \left(i_{k} \in I^{r e}\right) \\
\psi(\vec{x})-\psi_{k}\left(x_{k}+\sum_{k<j<k(+)}\left\langle h_{i_{k}}, \alpha_{i_{j}}\right\rangle x_{j}-x_{k(+)}\right) & \left(i_{k} \in I^{i m}\right)\end{cases} \\
& =\left(\widehat{S_{k}} \psi\right)(\vec{x}) \geq 0 \text {. }
\end{aligned}
$$

Now, suppose that $\tilde{e}_{i} \vec{x}$ does not satisfy the condition (ii). By (3.3) and (3.5), it is easy to see that $\tilde{e}_{i} \vec{x}$ satisfies (3.3). For instance, if $\tilde{e}_{i} \vec{x}\left(i \in I^{r e}\right)$ does not satisfy (3.3) $\left(t^{(-)}=0\right)$, then $x_{k}$ in $\vec{x}$ must be 1 , and

$$
\left.\left\langle h_{i_{t}}, \lambda\right\rangle=\left\langle h_{i_{t}}, \alpha_{i_{j}}\right\rangle x_{j}=0 \text { for all } j \neq k, 1 \leq j<t \quad \text { (in } \vec{x}\right) .
$$

That is, $\vec{x}$ satisfies the condition (3.4). Then by (3.5), we have $\widehat{S}_{k} x_{k}(\vec{x})=x_{k}-$ $\beta_{k}^{(+)}>0$, which implies $\beta_{k}^{(+)} \leq 0$. This contradicts the definition of the Kashiwara operator $\tilde{e}_{i}$. Moreover, by the same argument given in the proof of Theorem 3.1 in [19, it is proved that $\tilde{e}_{i} \vec{x}$ satisfies the condition (3.5). Therefore, $\Gamma_{\iota}$ is closed under all $\tilde{e}_{i}$.

To complete the proof, it suffices to show that if $\vec{x} \in \Gamma_{\iota}[\lambda]$ satisfies $\tilde{e}_{i} \vec{x}=0$ for any $i \in I$, then $\vec{x}=\overrightarrow{0}$. Suppose that $\tilde{e}_{i} \vec{x}=0$ for any $i \in I$ and $\vec{x} \neq \overrightarrow{0}$. Since $\vec{x} \neq \overrightarrow{0}$, we have $j>0$ such that $x_{j}>0$ and $x_{k}=0$ for all $k>j$. First, consider the case $i_{j} \in I^{r e}$. Since $\sigma_{j}(\vec{x})=x_{j}>0$, by (2.8), $\sigma^{(i)}(\vec{x})<\sigma_{0}^{(i)}(\vec{x})$ for $i_{j}=i$. Hence,

$$
0<\sigma_{0}^{(i)}(\vec{x})-\sigma^{(i)}(\vec{x}) \leq \sigma_{0}^{(i)}(\vec{x})-\sigma_{\iota^{(i)}}(\vec{x})=\beta_{\iota^{(i)}}^{(-)}(\vec{x})=-\lambda^{(-)}(\vec{x}) .
$$

This is a contradiction.

Second, consider the case that $i_{j} \in I^{i m}$. If $j^{(-)}=0$, by (2.8), we have

$$
\sigma_{0}^{(i)}(\vec{x})-a_{i i}=-\left\langle h_{i}, \lambda\right\rangle+\sum_{l \geq 1}\left\langle h_{i}, \alpha_{l}\right\rangle x_{l}-a_{i i} \geq 0,
$$

where $i_{j}=i$. But, by (3.3),

$$
\sigma_{0}^{(i)}(\vec{x})-a_{i i}=-\left\langle h_{i}, \lambda\right\rangle+\sum_{1 \leq l<j}\left\langle h_{i}, \alpha_{l}\right\rangle x_{l}+a_{i i}\left(x_{j}-1\right)<0 .
$$

This is a contradiction. Moreover, if $j^{(-)} \neq 0$, then $\sigma_{0}^{(i)}(\vec{x})-a_{i i}<0$ and

$$
x_{j}=1 \quad \text { and } \sum_{j^{(-)}<l<j}\left\langle h_{i_{j}}, \alpha_{i_{l}}\right\rangle x_{l}=0 .
$$

(Indeed, if $\sigma_{0}^{(i)}(\vec{x})-a_{i i} \geq 0$, we can derive a contradiction by the same argument given in the case that $j^{(-)}=0$.) But, this contradicts (3.3).

Now, recall the main result of polyhedral realization of $B(\infty)$ given in [19]. Let $S_{k}=S_{k, \iota}$ be a piecewise-linear operator on $\left(\mathbf{Q}^{\infty}\right)^{*}$ such that

$$
S_{k}(\psi)= \begin{cases}\psi-\psi_{k} \beta_{k} & \text { if } \psi_{k}>0, i_{k} \in I^{r e} \\ \psi-\psi_{k}\left(x_{k}+\sum_{k<j<k^{(+)}}\left\langle h_{i}, \alpha_{i_{j}}\right\rangle x_{j}-x_{k^{(+)}}\right) & \text {if } \psi_{k}>0, i_{k} \in I^{i m} \\ \psi-\psi_{k} \beta_{k^{(-)}} & \text {if } \psi_{k} \leq 0\end{cases}
$$

where $\beta_{k}=\beta_{k}^{(+)}$. Let

$$
\Theta_{\iota}=\left\{S_{j_{l}} \cdots S_{j_{1}} x_{j_{0}}: l \geq 0, j_{0}, \ldots, j_{l} \geq 1\right\}
$$


be the set of linear forms obtained from the coordinate forms $x_{j}$ by applying transformations $S_{k}$. Then under the positivity assumption on $\iota$ [18, 19], we have

Theorem 3.2 (19]). Let $\iota$ be the sequence of indices satisfying (2.5). Let $\Psi_{\iota}$ : $B(\infty) \hookrightarrow \mathbf{Z}_{\geq 0, \iota}^{\infty}$ be the Kashiwara embedding. Then $\operatorname{Im} \Psi_{\iota}$ is the set $\Gamma_{\iota}$ of $\vec{x} \in \mathbf{Z}_{\geq 0, \iota}^{\infty}$ such that

(i) $\psi(\vec{x}) \geq 0$ for any $\psi \in \Theta_{\iota}$,

(ii) for each $t$ with $i_{t} \in I^{i m}$, if $x_{t} \neq 0$ and $t^{(-)} \neq 0$, then

$$
\sum_{t^{(-)}<j<t}\left\langle h_{i_{t}}, \alpha_{i_{j}}\right\rangle x_{j}<0 .
$$

In addition, if $\left\langle h_{i_{t}}, \alpha_{i_{j}}\right\rangle x_{j}=0\left(t^{(-)}<j<t\right)$ for all $j$ with $i_{j} \in I^{i m}$, there exists a $p\left(t^{(-)}<p<t\right)$ such that $i_{p} \in I^{r e}$,

$$
\left\langle h_{i_{t}}, \alpha_{i_{p}}\right\rangle x_{p}<0 \text { and } \psi(\vec{x})>0 \text { for any } \psi \in \Theta_{\iota}^{p \backslash t} \text {. }
$$

Corollary 3.3 (19]). Assume that all elements of $I$ are imaginary, that is, $I=$ $I^{i m}$. Then the image of the Kashiwara embedding $\operatorname{Im} \Psi_{\iota}$ equals the set of $\vec{x} \in \mathbf{Z}_{\geq 0, \iota}^{\infty}$ satisfying (3.6) of Theorem 3.2 .

Corollary 3.4 (19). Let $I$ be an index set such that the cardinality of $I^{\text {re }}$ is 1 , and let $\iota$ be a sequence of indices in I satisfying (2.5). Then the image $\operatorname{Im} \Psi_{\iota}$ of the crystal embedding is the set $\Gamma_{\iota}$ of $\vec{x} \in \mathbf{Z}_{\geq 0, \iota}^{\infty}$ satisfying the following conditions:

(i) $S_{j} x_{j} \geq 0$ for all $j$ with $i_{j} \in I^{\text {re }}$,

(ii) for each $t$ with $i_{t} \in I^{\text {im }}$, if $x_{t} \neq 0$ and $t^{(-)} \neq 0$, then

$$
\sum_{t^{(-)}<j<t}\left\langle h_{i_{t}}, \alpha_{i_{j}}\right\rangle x_{j}<0 .
$$

In addition, if $\left\langle h_{i_{t}}, \alpha_{i_{j}}\right\rangle x_{j}=0\left(t^{(-)}<j<t\right)$ for all $i_{j} \in I^{i m}$, then there exists a $p\left(t^{(-)}<p<t\right)$ such that $i_{p} \in I^{r e}$,

$$
\left\langle h_{i_{t}}, \alpha_{i_{p}}\right\rangle x_{p}<0 \text { and } S_{p} x_{p}>0 \text {. }
$$

Now, define a linear form $\xi^{(i)}\left(i \in I^{r e}\right)$ on $\mathbf{Q}^{\infty}$ by

$$
\xi^{(i)}(\vec{x})=-\sum_{1 \leq j<\iota(i)}\left\langle h_{i}, \alpha_{i_{j}}\right\rangle x_{j}-x_{\iota}^{(i)}=-\left\langle h_{i}, \lambda\right\rangle+\lambda^{(i)}(\vec{x}),
$$

and set

$$
\Theta_{\iota}^{(i)}=\left\{S_{j_{l}} \ldots S_{j_{1}} \xi^{(i)} \mid l \geq 0, j_{1}, \ldots, j_{l} \geq 1\right\} .
$$

Then the strict positivity assumption for $\iota$ [17] is as follows:

$$
\begin{gathered}
\text { for any } \psi=\sum_{k} \psi_{k} x_{k} \in\left(\Theta_{\iota} \cup\left(\bigsqcup_{j \in I} \Theta_{\iota}^{j}\right)\right) \backslash\left\{\xi^{(j)} \mid j \in I\right\}, \\
\psi_{k} \geq 0 \text { if } k^{(-)}=0 .
\end{gathered}
$$

Proposition 3.5. For the sequence $\iota$ satisfying the strict positivity assumption, we have

$$
\widehat{S}_{j_{l}} \ldots \widehat{S}_{j_{1}} x_{j_{0}}=S_{j_{l}} \ldots S_{j_{1}} x_{j_{0}}
$$

for any $l \geq 0, j_{0}, \ldots, j_{l} \geq 1$, and

$$
\widehat{S}_{j_{l}} \ldots \widehat{S}_{j_{1}} \lambda^{(i)}(\vec{x})=\left\langle h_{i}, \lambda\right\rangle+S_{j_{l}} \ldots S_{j_{1}} \xi^{(i)}(\vec{x})
$$

for any $l \geq 0, j_{1}, \ldots, j_{l} \geq 1$, if the left hand side of (3.8) is nonzero. 
Proof. By the same argument as in the proof of Lemma 4.3 in [17, it is proved. So we omit it.

By Proposition 3.5. we know that under the strict positivity assumption for $\iota$, the set $\Gamma_{\iota}[\lambda]\left(\lambda \in P^{+}\right)$has $\overrightarrow{0}$. Moreover, we have

Corollary 3.6. Let $\iota$ be a sequence of indices satisfying (2.5) and $\lambda$ be a dominant integral weight. Suppose that $\overrightarrow{0}$ belongs to $\Gamma_{\iota}[\lambda]$. Then we have

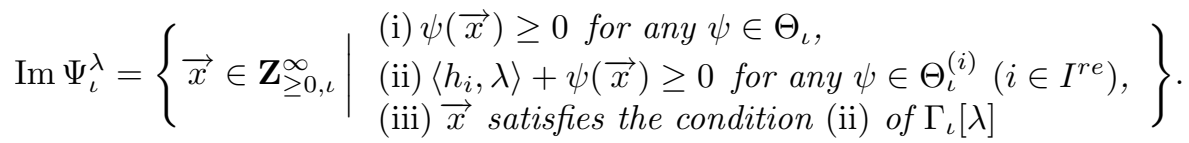

Corollary 3.7. Assume that all elements of $I$ are imaginary, that is, $I=I^{i m}$. Then the image of the Kashiwara embedding $\operatorname{Im} \Psi_{\iota}^{\lambda}$ equals the set of $\vec{x} \in \mathbf{Z}_{\geq 0, \iota}^{\infty}[\lambda]$ satisfying (3.3) of Theorem 3.1.

Proof. By Corollary 3.6, it suffices to consider the set $\Theta_{\iota}^{(i)}$. By a simple calculation, it is easy to see that the set $\Theta_{\iota}^{(i)}$ consists of the linear combinations of the coordinate forms $x_{j}$ with nonnegative coefficients, which completes the proof.

By Corollary 3.4 and Corollary [3.6, we have the following simple but important corollary.

Corollary 3.8. Let $I$ be an index set such that the cardinality of $I^{r e}$ is 1 , and let $\iota$ be a sequence of indices in I satisfying (2.5). Then the image $\operatorname{Im} \Psi_{\iota}^{\lambda}$ of the crystal embedding is the set $\Gamma_{\iota}[\lambda]$ of $\vec{x} \in \mathbf{Z}_{\geq 0, L}^{\infty}[\lambda]$ satisfying the following conditions:

(i) $S_{j} x_{j} \geq 0$ for all $j$ with $i_{j} \in I^{r e}$,

(ii) $\left\langle h_{i}, \lambda\right\rangle+\psi(\vec{x}) \geq 0$ for any $\psi \in \Theta_{\iota}^{(i)}\left(i \in I^{r e}\right)$,

(iii) for each $t$ with $i_{t} \in I^{i m}$, if $x_{t} \neq 0$, then

$$
\begin{aligned}
\sum_{t^{(-)}<j<t}\left\langle h_{i_{t}}, \alpha_{i_{j}}\right\rangle x_{j}<0 & \left(t^{(-)} \neq 0\right), \\
-\left\langle h_{i_{t}}, \lambda\right\rangle+\sum_{1 \leq j<t}\left\langle h_{i_{t}}, \alpha_{i_{j}}\right\rangle x_{j}<0 & \left(t^{(-)}=0\right) .
\end{aligned}
$$

In addition, if

$$
\begin{aligned}
& \sum_{\substack{t(-)<j<t \\
j \in I^{i m}}}\left\langle h_{i_{t}}, \alpha_{i_{j}}\right\rangle x_{j}=0\left(t^{(-)} \neq 0\right), \\
&-\left\langle h_{i_{t}}, \lambda\right\rangle+\sum_{\substack{1 \leq j<t \\
j \in I^{i m}}}\left\langle h_{i_{t}}, \alpha_{i_{j}}\right\rangle x_{j}=0 \quad\left(t^{(-)}=0\right),
\end{aligned}
$$

then there exists a $p\left(t^{(-)}<p<t\right)$ with $i_{p} \in I^{r e}$ such that

$$
\left\langle h_{i_{t}}, \alpha_{i_{p}}\right\rangle x_{p}<0 \text { and } \widehat{S}_{p} x_{p}(\vec{x})>0 .
$$

Set $W(\lambda)=\left\{\mu \in P \mid B(\lambda)_{\mu} \neq \emptyset\right\}$ and denote by $m_{\lambda, \mu}$ the weight multiplicity of $\mu$ in $B(\lambda)$. Any $\mu \in W(\lambda)$ is written as $\lambda-\sum_{i} m_{i} \alpha_{i}$ for $m_{i} \in \mathbf{Z}_{\geq 0}$.

Corollary 3.9. For $\mu=\lambda-\sum_{i} m_{i} \alpha_{i} \in W(\lambda)$, the weight multiplicity of $\mu$ is given by

$$
m_{\lambda, \mu}=\#\left\{\vec{x} \in \Gamma_{\iota}[\lambda] \mid m_{i}=\sum_{i_{k}=i} x_{k} \text { for any } i \in I\right\} .
$$




\section{Applichtions: Monster Lie algebras}

In this section, we will give an explicit description of the image of the Kashiwara embedding for the generalized Kac-Moody algebras of ranks 2, 3 and Monster Lie algebras. First, consider the rank 2 case. Assume that $I=\{1,2\}$ and $\iota=$ $(\ldots, 2,1,2,1)$. Since we have the results for the Kac-Moody case [17, 18, we also assume that one of $1,2 \in I$ is imaginary. Thanks to Corollary 3.3] it is enough to consider the case that $1 \in I^{i m}$ and $2 \in I^{r e}$. Set

$$
\left\langle h_{1}, \alpha_{1}\right\rangle=-a,\left\langle h_{1}, \alpha_{2}\right\rangle=-b,\left\langle h_{2}, \alpha_{1}\right\rangle=-c, \text { and }\left\langle h_{2}, \alpha_{2}\right\rangle=2,
$$

where $a, b, c \in \mathbf{Z}_{\geq 0}$.

Corollary 4.1. Let $\lambda$ be a dominant integral weight. Assume that $1 \in I^{i m}$ and $2 \in I^{r e}$. The image of the Kashiwara embedding $\operatorname{Im} \Psi_{\iota}^{\lambda}$ is given by the subset $\Gamma_{\iota}[\lambda]$ of $\vec{x} \in \mathbf{Z}_{\geq 0, \iota}^{\infty}[\lambda]$ as follows:

(a) When $b=c=0$,

(i) $x_{k}=0$ for $k \geq 3$,

(ii) $-x_{2}+\left\langle h_{2}, \lambda\right\rangle \geq 0$,

(iii) $x_{1} \neq 0$ implies $\left\langle h_{1}, \lambda\right\rangle>0$.

(b) When neither $b$ nor $c$ is 0 ,

(i) $c x_{1}-x_{2}+\left\langle h_{2}, \lambda\right\rangle \geq 0$,

(ii) for each $k \geq 1$, cx $x_{2 k+1}-x_{2 k+2}>0$ unless $x_{2 k+1}=x_{2 k+2}=0$,

(iii) for each $k \geq 1$, if $x_{2 k+1} \neq 0$, then $x_{2 k}>0$,

(iv) $x_{1} \neq 0$ implies $\left\langle h_{1}, \lambda\right\rangle>0$.

Proof. By Example 3.4 in 19 and Corollary [3.8, it suffices to consider

$$
\xi^{(2)}=-\sum_{1 \leq j<2}\left\langle h_{2}, \alpha_{i_{j}}\right\rangle x_{j}-x_{2}=c x_{1}-x_{2} \text { and } S_{j_{l}} \ldots S_{j_{1}} \xi^{(2)} .
$$

Since $S_{j_{l}} \ldots S_{j_{1}} \xi^{(2)}$ is a linear combination of $x_{i}$ 's with nonnegative coefficients, the only meaningful element is $c x_{1}-x_{2}$. Moreover, if $x_{1}$ is nonzero, by (3.3), we have $\left\langle h_{1}, \lambda\right\rangle>0$.

Example 4.2. If $b=1, c=2$, and $\left\langle h_{1}, \lambda\right\rangle=\left\langle h_{2}, \lambda\right\rangle=1$, then the top part of $\operatorname{Im} \Psi_{\iota}^{\lambda} \cong B(\lambda)$ is as follows:

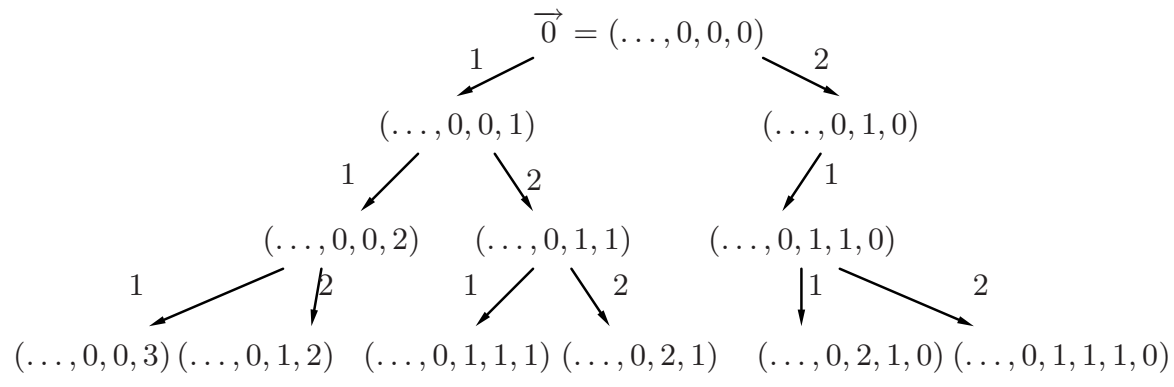


Now, let us apply Corollary 3.8 to the generalized Kac-Moody algebras of rank 3 . Assume that $I=\{1,2,3\}$ and $\iota=(\ldots, 1,3,2,1)$. Assume that $1,2 \in I^{i m}, 3 \in I^{r e}$. Let $A$ be a Borcherds-Cartan matrix

$$
A=\left(\begin{array}{rrr}
-a & -b & -c \\
-d & -e & -f \\
-g & -h & 2
\end{array}\right),
$$

where $a, b, c, d, e, f, g, h \in \mathbf{Z}_{\geq 0}$.

Corollary 4.3. Let $\lambda$ be a dominant integral weight. The image of the Kashiwara embedding $\operatorname{Im} \Psi_{\iota}^{\lambda}$ is given by the subset $\Gamma_{\iota}[\lambda]$ of $\vec{x} \in \mathbf{Z}_{\geq 0, \iota}^{\infty}[\lambda]$ satisfying the following conditions:

(i) $g x_{1}+h x_{2}-x_{3}+\left\langle h_{3}, \lambda\right\rangle \geq 0$,

(ii) for each $k \geq 1$, gx $x_{3 k+1}+h x_{3 k+2}-x_{3 k+3} \geq 0$,

(iii) for each $k \geq 4$ with $i_{k}=1,2$, if $x_{k} \neq 0$, then

$$
\begin{gathered}
b x_{k-2}+c x_{k-1}>0\left(i_{k}=1\right), \quad f x_{k-2}+d x_{k-1}>0\left(i_{k}=2\right) . \\
\text { Moreover, if }\left\langle h_{i_{k}}, \alpha_{i_{j}}\right\rangle x_{j}=0 \text { for } j=k-1, k-2 \text { with } i_{j} \neq 3, \text { then } \\
g x_{j+1}+h x_{j+2}-x_{j+3}>0 .
\end{gathered}
$$

(iv) $x_{1} \neq 0$ and $x_{2} \neq 0$ imply

$$
\left\langle h_{1}, \lambda\right\rangle>0 \text { and }-\left\langle h_{2}, \lambda\right\rangle-d x_{1}<0 \text {, respectively. }
$$

Proof. By Theorem 7 in [19], and Corollary [3.6, it suffices to consider the set

$$
\Theta_{\iota}^{(i)}=\left\{S_{j_{l}} \ldots S_{j_{1}} \xi^{(i)} \mid l \geq 0, j_{1}, \ldots, j_{l} \geq 1\right\} .
$$

We know that $\xi^{(3)}=g x_{1}+h x_{2}-x_{3}$ and

$$
\begin{aligned}
& S_{1}\left(\xi^{(3)}\right)=(b g+h) x_{2}+(c g-1) x_{3}+g x_{4}, \\
& S_{2}\left(\xi^{(3)}\right)=g x_{1}+(f h-1) x_{3}+d h x_{4}+h x_{5} .
\end{aligned}
$$

Since $S_{1}\left(\xi^{(3)}\right)$ and $S_{2}\left(\xi^{(3)}\right)$ are linear combinations with nonnegative coefficients of $x_{i}$ 's, it is clear that $S_{j_{l}} \ldots S_{j_{1}} \xi^{(i)} \geq 0(l \geq 1)$ for all $\vec{x} \in \Gamma_{\iota}$.

Let $I=\left\{-1=-1_{1}\right\} \cup\left\{i_{t} \mid i \in \mathbf{N}, t=1, \ldots, c(i)\right\}$, where $c(i)$ is the coefficient of the elliptic modular function

$$
j(q)-744=q^{-1}+196884 q+21493760 q^{2}+\cdots=\sum_{i=-1}^{\infty} c(i) q^{i} .
$$

We define $A=\left(a_{p q}\right)_{p, q \in I}$ to be the matrix such that

$$
a_{p q}=-(i+j) \quad \text { if } p=i_{l}, q=j_{m} \text { for } 1 \leq l \leq c(i), 1 \leq m \leq c(j) .
$$

The associated generalized Kac-Moody algebra $\mathfrak{g}$ is called the Monster Lie algebra, and it played a crucial role in Borcherds' proof of the Moonshine conjecture 2]. More precisely, Borcherds derived the twisted denominator identity for the Monster Lie algebra with the action of the Monster, from which the replication formulae for the Thompson series follow.

In this paper, we deal with the corresponding quantum group $U_{q}(\mathfrak{g})$, which we call the quantum Monster algebra. Assume that

$$
\begin{array}{r}
\iota=\left(\ldots,-1,3_{c(3)}, \ldots, 3_{1}, 2_{c(2)}, \ldots, 2_{1}, 1_{c(1)}, \ldots, 1_{1},-1,\right. \\
\left.2_{c(2)}, \ldots, 2_{1}, 1_{c(1)}, \ldots, 1_{1},-1,1_{c(1)}, \ldots, 1_{1},-1\right) .
\end{array}
$$


Let $I_{(-1)}$ be the set of positive integers $k$ such that $i_{k}=-1$; i.e.,

$$
I_{(-1)}=\{1\} \cup\{b(n)=n c(1)+(n-1) c(2)+\cdots+c(n)+n+1 \mid n \in \mathbf{N}\},
$$

and for any $n \geq 1$,

$$
\sigma(n)=c(1)+\cdots+c(n) .
$$

Theorem 4.4. Let $\lambda$ be a dominant integral weight. Then the image of the Kashiwara embedding $\operatorname{Im} \Psi_{\iota}^{\lambda}$ is given by the subset $\Gamma_{\iota}[\lambda]$ of $\vec{x} \in \mathbf{Z}_{\geq 0, \iota}^{\infty}[\lambda]$ such that

(i) $0 \leq x_{1} \leq\left\langle h_{-1}, \lambda\right\rangle, x_{c(1)+2}=0$, and for each $n \geq 1$,

$$
\sum_{k=1}^{n} k\left(x_{b(n)+\sigma(k)+1}+\cdots+x_{b(n)+\sigma(k+1)}\right)-x_{b(n)+\sigma(n+1)+1} \geq 0,
$$

(ii) for each $k \notin I_{(-1)}$, if $x_{k} \neq 0$ and $k^{(-)} \neq 0$, then

$$
\sum_{k^{(-)}<j<k}\left\langle h_{i_{k}}, \alpha_{i_{j}}\right\rangle x_{j} \neq 0
$$

Moreover, if $\left\langle h_{i_{k}}, \alpha_{i_{j}}\right\rangle x_{j}=0$ for all $k^{(-)}<j<k$ with $j \notin I_{-1}$, then there exists $m \geq 1$ such that $k^{(-)}<b(m)<k$ and

$$
\sum_{k=1}^{m} k\left(x_{b(m)+\sigma(k)+1}+\cdots+x_{b(m)+\sigma(k+1)}\right)-x_{b(m)+\sigma(m+1)+1}>0,
$$

(iii) for each $k \notin I_{-1}$, if $x_{k} \neq 0$ and $k^{(-)}=0$, then

$$
-\left\langle h_{i_{k}}, \lambda\right\rangle+\sum_{1 \leq j<k}\left\langle h_{i_{k}}, \alpha_{i_{j}}\right\rangle x_{j}<0 .
$$

Moreover, if $\left\langle h_{i_{k}}, \lambda\right\rangle=0$ and $\left\langle h_{i_{k}}, \alpha_{i_{j}}\right\rangle x_{j}=0$ for all $1 \leq j<k$ with $j \notin I_{-1}$, then there exists $m \geq 1$ such that $1 \leq b(m)<k$ and

$$
\sum_{k=1}^{m} k\left(x_{b(m)+\sigma(k)+1}+\cdots+x_{b(m)+\sigma(k+1)}\right)-x_{b(m)+\sigma(m+1)+1}>0 .
$$

Proof. By Theorem 10 in [19] and Corollary [3.6] it is enough to consider the set

$$
\Theta_{\iota}^{(i)}=\left\{S_{j_{l}} \ldots S_{j_{1}} \xi^{(i)} \mid l \geq 0, j_{1}, \ldots, j_{l} \geq 1\right\} .
$$

We know that $\xi^{(-1)}=-x_{1}$ and $S_{j_{l}} \ldots S_{j_{1}} \xi^{(i)}=-x_{1}$ for all $l$, which completes the proof.

Finally, by Theorem 4.4 we have the following character of the highest weight module $V(\lambda)$ over $U_{q}(\mathfrak{g})$.

\section{Corollary 4.5.}

$$
\operatorname{ch} V(\lambda)=\sum_{\vec{x} \in \Gamma_{\iota}[\lambda]} e^{\mathrm{wt}(\vec{x})}=\sum_{\vec{x} \in \Gamma_{\iota}[\lambda]} e^{\lambda-\sum_{j=1}^{\infty} x_{j} \alpha_{i_{j}}} .
$$

\section{ACKNOWLEDGMENTS}

The author would like to express his sincere gratitude to Professor S.-J. Kang and Professor J.-A. Kim for their interest in this work and many valuable discussions. 


\section{REFERENCES}

[1] R. E. Borcherds, Generalized Kac-Moody algebras, J. Algebra 115 (1988), 501-512. MR.943273 (89g:17004)

[2] R. E. Borcherds, Monstrous moonshine and monstrous Lie superalgebras, Invent. Math. 109 (1992), 405-444. MR.1172696 (94f:11030)

[3] G. Cliff, Crystal bases and Young tableaux, J. Algebra 202 (1998), 10-35. MR.1614241 (99k:17025)

[4] J. H. Conway, S. Norton, Monstrous moonshine, Bull. London Math. Soc. 11 (1979), 308-339. MR554399 (81j:20028)

[5] V. G. Drinfel'd, Hopf algebras and the quantum Yang-Baxter equation, Soviet Math. Dokl., 32 (1985), 254-258. MR802128 (87h:58080)

[6] M. Jimbo, A q-difference analogue of $U(\mathfrak{g})$ and the Yang-Baxter equation, Lett. Math. Phys. 10 (1985), 63-69. MR797001 (86k:17008)

[7] K. Jeong, S.-J. Kang, M. Kashiwara, Crystal bases for quantum generalized Kac-Moody algebras, Proc. London Math. Soc. 90 (2005), 395-438. MR2142133 (2006e:17020)

[8] K. Jeong, S.-J. Kang, M. Kashiwara, D.-U. Shin, Abstract crystals for quantum generalized Kac-Moody algebras, Int. Math. Res. Not. IMRN 2007, Art. ID mm001, 19 pp. MR2331899

[9] S.-J. Kang, Quantum deformations of generalized Kac-Moody algebras and their modules, J. Algebra 175 (1995), 1041-1066. MR1341758 (96k:17023)

[10] S.-J. Kang, Crystal bases for quantum affine algebras and combinatorics of Young walls, Proc. London Math. Soc. 86 (2003), 29-69. MR.1971463 (2004c:17028)

[11] S.-J. Kang, M. Kashiwara, K. C. Misra, T. Miwa, T. Nakashima, A. Nakayashiki, Affine crystals and vertex models, Adv. Ser. Math. Phys., vol. 16, World Sci. Publ., River Edge, NJ, 1992, pp. 449-484. MR 1187560 (94a:17008)

[12] M. Kashiwara, Crystalizing the q-analogue of universal enveloping algebras, Comm. Math. Phys. 133 (1990), 249-260. MR1090425 (92b:17018)

[13] M. Kashiwara, On crystal bases of the q-analogue of universal enveloping algebras, Duke Math. J. 63 (1991), 465-516. MR.1115118 (93b:17045)

[14] M. Kashiwara, The crystal base and Littelmann's refined Demazure character formula, Duke Math. J. 71 (1993), 839-858. MR.1240605 (95b:17019)

[15] M. Kashiwara, T. Nakashima, Crystal graphs for representations of the q-analogue of classical Lie algebras, J. Algebra 165 (1994), 295-345. MR.1273277 (95c:17025)

[16] P. Littelmann, Paths and root operators in representation theory, Ann. of Math. 142 (1995), 499-525. MR1356780(96m:17011)

[17] T. Nakashima, Polyhedral realizations of crystal bases for integrable highest weight modules, J. Algebra 219 (1999), 571-597 MR:1706829 (2000g:17020)

[18] T. Nakashima, A. Zelevinsky, Polyhedral realizations of crystal bases for quantized KacMoody algebras, Adv. Math. 131 (1997), 253-278 MR1475048 (98m:17023)

[19] D.-U. Shin, Polyhedral realization of crystal bases for generalized Kac-Moody algebras, J. London Math. Soc. (2) 77 (2008), 273-286.

Department of Mathematics Education, Hanyang University, Seoul 133-791, Korea

E-mail address: dushin@hanyang.ac.kr 